\title{
Web-based actuator selection tool
}

\author{
John D. Madden* and Luca Filipozzi, Department of Electrical \& Computer Engineering \\ Advanced Materials and Process Engineering Laboratory, University of British Columbia, \\ Vancouver, BC, Canada V6T 1 Z4
}

\begin{abstract}
Device designers are continually confronted with the challenge of selecting the best actuator for a task and developers of new actuators are seeking applications for which their technologies are suitable. A web-based interface is presented that enables designers to input basic needs (force, displacement, frequency, cycle life, dimensions, voltage and power available) and retrieve an initial evaluation of the suitability of the various actuator technologies in the database. The prototype contains data for a number of emerging technologies including conducting polymers, dielectric elastomers, ferroelectric polymers, thermal and magnetic shape memory alloys, carbon nanotube actuators, liquid crystal elastomers, ionic polymer metal composites and mammalian skeletal muscle. The system is very early in the development process, and it is hoped that feedback from the EAP community will help guide the growth of and establish the need for this tool.
\end{abstract}

Keywords: Conducting polymers, dielectric elastomers, ferroelectric polymers, SMA, carbon nanotube actuators, liquid crystal elastomers, IPMC.

\section{INTRODUCTION}

A simple approach is presented to enable actuator comparison. The comparison algorithm is reproduced online at http://mm.ece.ubc.ca/actuators. The approach assumes that the designer has in mind particular actuator dimensions, the approximate force and displacement required, the number of cycles, the rate of actuation and any constraints on applied potential. It is well suited for situations in which a linear displacement is needed, and is designed to compare linear actuators. The algorithm then determines which actuator technologies are able to generate sufficient work output within the available volume in a single actuator stroke. Actuator technologies are also compared based on the amount of mechanical amplification needed to produce the desired displacement. Actuators having sufficient power are also identified. In some cases actuators have sufficient power but cannot perform the full work required in a single stroke. In such cases it may be possible to perform multiple actuator strokes per cycle (e.g. as in a ratchet mechanism). The number of strokes per cycle is estimated for such cases. Finally actuators can be selected based on their mass, and the mass of the power source employed.

The approach to actuator selection follows the basic algorithm presented in recent papers ${ }^{3-5}$. In these cases a set of initial product specifications is used as the basis for justifying actuator selection. A comprehensive tool for comparing a wide range of actuator technologies, which includes commercial actuator specifications, is available ${ }^{9}$. The approach here is much more limited in its scope, but covers emerging materials that are often not represented or up to date in the commercial package and is also intended to be very simple to use.

Along with a review article used as the basis of the work presented here ${ }^{4}$, a number of other sources for obtaining detailed information on actuators are recommended ${ }^{1 ; 2 ; 6-8}$.

\subsection{Limitations}

The tool is very crude and is only intended to provide an order of magnitude estimate of feasibility of an application. It is also intended to give relatively optimistic assessments of the prospects for each actuator technology. More detailed analysis needs to follow, including reading of the actuator literature and consultation or collaboration with experts. Few of the actuators presented are available off the shelf and often the needed specifications are not known (e.g. temperature dependence of operation is only published for a few actuators). Those that are available off the shelf come in a very limited range of geometries. As a result designers who identify candidate actuator technologies for their application should anticipate some research and development work.

*jmadden@ece.ubc.ca, www.ece.ubc.ca/ jmadden. 
A real challenge in comparing actuator technologies is that figures of merit are seldom independent. For example, actuator strain and work density are functions of load and cycle number. Eventually as models of these inter-

relationships evolve they may be incorporated into a comparison database, but at present they are not. For this reason calculations performed are approximate at best. Limitations specific to each calculation performed by the web page are discussed in more detail below.

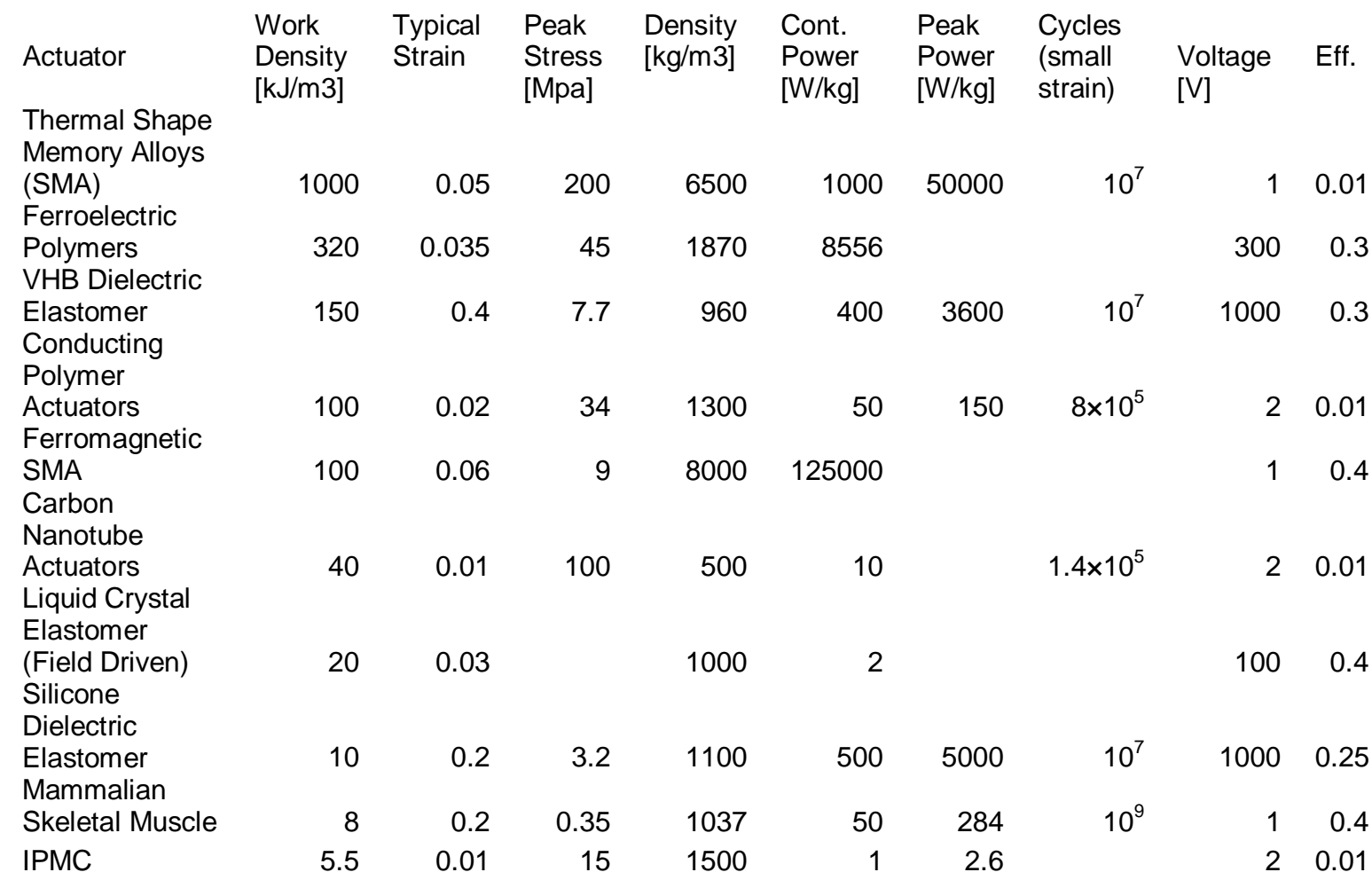

Table 1: The actuator technologies investigated and the data used to compare their properties. Eff. is an abbreviation of efficiency, and cont. is an abbreviation of continuous.

\subsection{Data}

The actuator properties that are initially used in the web tool are selected from a review article ${ }^{4}$ and presented in Table 1. Performance metrics can change dramatically with time. In carbon nanotube actuators the load carrying capability increased by more than an order of magnitude within one year, for example. We hope that the community will provide updated information so that actuators are fairly represented. The data table can be downloaded from the web site by scrolling to the end of the page and selecting "data" under the heading "Other Resources".

The actuator properties used in calculations at this point are:

- Actuator Work Density, which is the typical work performed per unit actuator volume. This volume does not include the volume of any required solvent, encapsulation, contacts and pre-stretching apparatus.

- Typical actuator strain is the strain that is readily achieved under a normal load.

- Density is the mass per unit volume of the actuator.

- (Specific) c)ontinuous powe) is the average power output per stroke per unit actuator mass under typical operating conditions.

- $\quad$ Cycles are the maximum number of cycles achieved as reported in the literature. Note that this is not necessarily achieved for cycles performed under the average strain. In many cases cycle life has not been fully characterized and the number shown is a lower bound.

- Efficiency is the ratio of work output divided by input energy that can be achieved under typical actuation conditions. In some cases the efficiency value assumes that some energy can be recovered. 


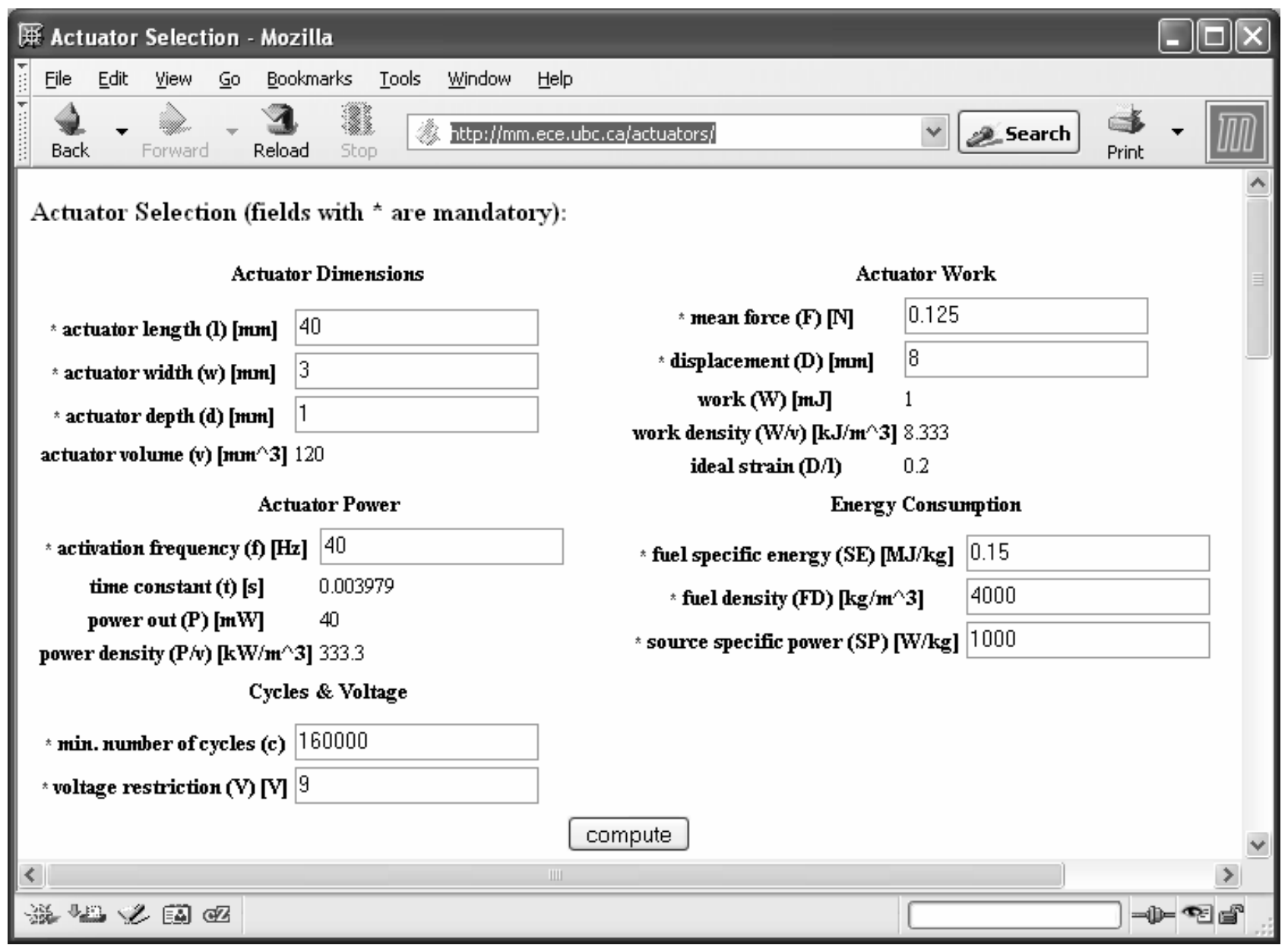

Figure 1: Inputs to the comparison tool (in the text boxes) and the calculated quantities.

\section{METHODS}

\subsection{Inputs}

The web page is shown in Figure 1. The inputs are:

- Dimensions

○ Length, $l$, width, $w$ and depth, $d$ representing the dimensions of the space available to the actuator. Length, $l$, is generally assumed to be the direction in which displacement is to be performed.

- $\quad$ Actuator Work

○ Force, $F$, and displacement, $d$ are the average force and displacement needed.

- Actuator Power

- Frequency, $f$ of operation is the maximum frequency at which the actuator is expected to operate. If time constant, $\tau$ is know it can be related to frequency via $f=(2 \pi \tau)^{-1}$.

- Cycles and Voltage

- Cycles, $c$ represents the number of times the force and displacement need to be applied.

$\circ$ Voltage, $V$ is the maximum voltage allowable. This may be due to regulatory restrictions or simply the desire to avoid voltage step-up.

- Energy Consumption: This section is useful if a portable power source is being employed.

- Fuel specific energy, $S E$, is the energy per unit mass of the power source (e.g. a battery or fuel cell).

- Fuel density, $F D$, is the mass per unit volume of the power source. (Not used currently.)

- Source specific power, $S P$, is the amount of power per unit mass of the energy source. 
At present all inputs are required. They are used in a number of calculations as discussed below.

\subsection{Output variables}

The model outputs a number of values, as shown in figure 1 . These are:

- Actuator volume, $v$, which is simply the product of $l \times w \times d$.

- The work, $W=F \times D$.

- The minimum work density, $W D=W / v$. If an actuator is to perform the required work within the specified volume in one stroke its work density must exceed this value.

- Ideal strain, $I S$, is the displacement, $D$, divided by the length, $l$. If an actuator is capable of such a strain then no mechanical amplification is required.

- Time constant, $t$, is the time constant associated with the input frequency. It is not currently used in any further calculations or plots.

- $\quad$ Power out, $P$, is the average power output per cycle, given by $P=W^{*} f$.

- Power density, $P D$, is the average power per unit volume, given by $P / v$. It is the minimum power per unit volume necessary to meet the specified work and frequency requirements.

\subsection{Plotted quantities}

The plots that are generated are shown in Figure 2. Below is a description of each plot that can also be obtained by clicking "help" on the web page immediately beneath the plots. The underlined quantities in the equations are from Table 1.

\subsubsection{Graph 1: Normalized Work Density vs. Mechanical Amplification}

This plot shows how much work each actuator can perform per stroke relative to the minimum work density needed for the application (Y-axis), and the amount of mechanical amplification required to achieve the needed displacement (Xaxis).

- $\quad$ Relative Work Density:

- Minimum Work Density $W D=W / v$

- Relative work density $=\underline{\text { Actuator Work Density }} / W D$

- Mechanical Amplification

- Mechanical amplification $=$ Actuator strain $/ I S$

Here Actuator Work Density and Actuator Strain are from the data table. If relative work density is greater than 1 then the actuator is capable of performing the required work, $W$ in one cycle. A horizontal red line indicates the divide between acceptable and unacceptable performance. If an actuator is deemed unable to perform the work in a single cycle, but is able to do it in multiple strokes per cycle because it is has sufficient power, then the work density restriction is no longer considered and the actuator is not stricken from the list of candidates printed at the bottom of the web page.

The relative work density likely needs to be at least 2 in order for it to be practical to consider achieving the work in one stroke. This is because only the actuator volume is considered in the calculation, and not any needed electrolyte, contacts, amplification mechanisms and the like.

\subsubsection{Graph 2: Power Density}

Which actuators have sufficient power per unit volume to perform the task? Those above the red line are deemed to have sufficient power because their continuous power density is higher than $P D=P / v_{2}$ the minimum continuous power density. Continuous specific power is multiplied by density to obtain the continuous power density, which is then plotted in graph 2 . The power density likely needs to be at least $2 \times P D$ in order for it to be practically achievable. This is because only the actuator volume is considered in the calculation, and not any needed electrolyte, contacts, amplification mechanisms and the like. 


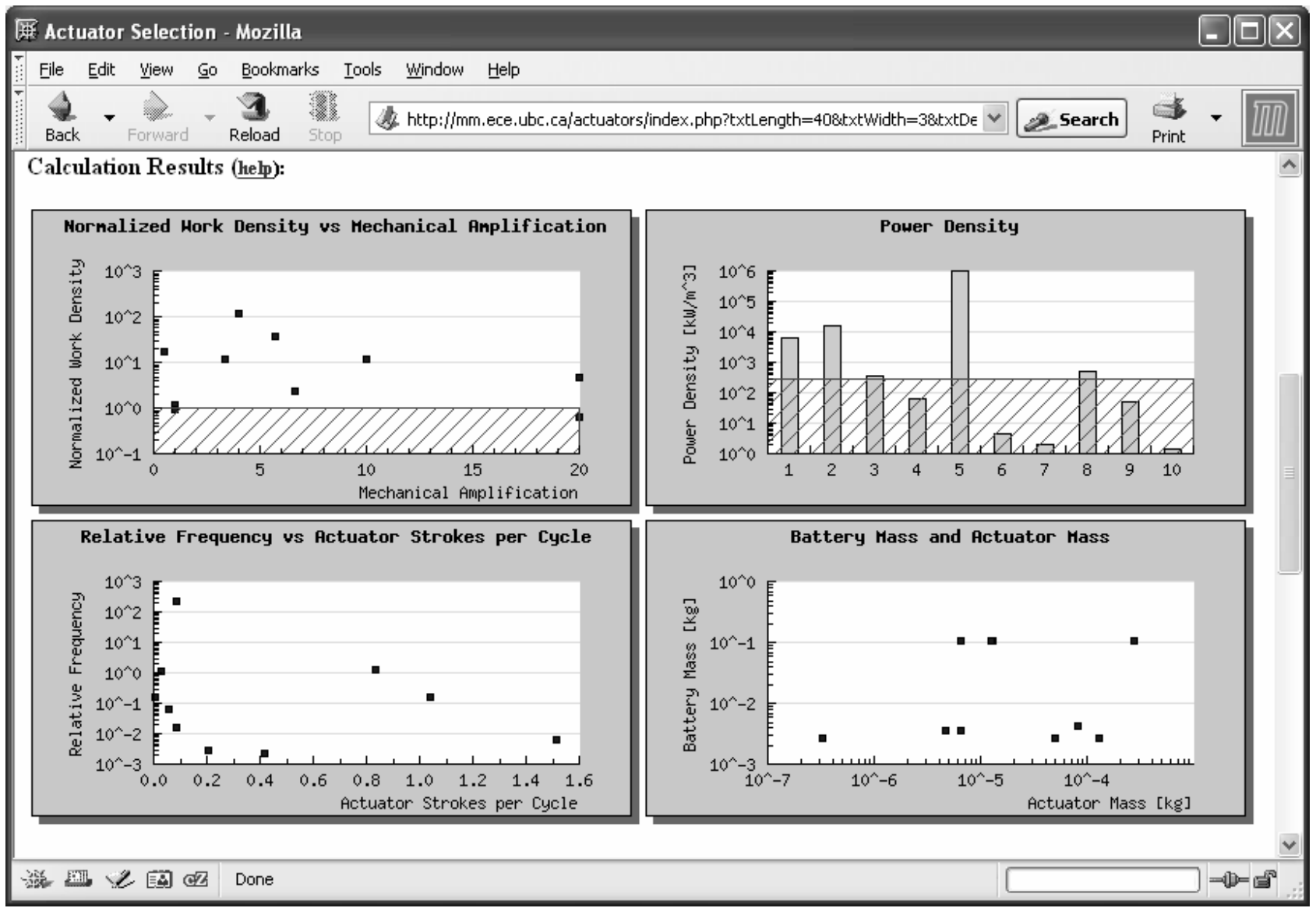

Figure 2: The plots obtained based on the inputs and the actuator data.

\subsubsection{Graph 3: Relative frequency vs. strokes per cycle.}

Some actuators can perform the work for one cycle in many strokes (e.g. inchworms, or a rotary electric motor driving a linear stage). The minimum number of strokes per cycle, $S P C=W D /$ Actuator work density. The larger the number of strokes per cycle, often the more complex the transmission mechanism that is required.

For the multiple strokes per cycle to succeed, the actuator must be capable of working at a frequency of $S P C x f$. Very little data is currently available on frequency response. The actuator bandwidth, $f_{b}$, (max frequency at which

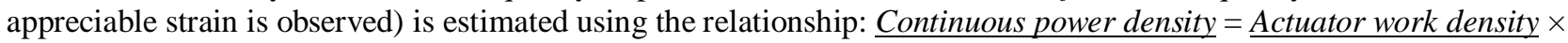
$f b$, where continuous power density is continuous specific power is multiplied by density. Relative frequency is $f b / f$. In order for a particular actuator technology to be capable of performing the work in multiple cycles, $f b / f \geq S P C$.

\subsubsection{Graph 4: Mass}

This plot estimates the masses of actuators and of the power source needed to run each actuator. The minimum actuator mass (X-axis) is Actuator volume $\times$ density where either:

$$
\text { Actuator volume }=v \times W D / \underline{\text { Actuator Work Density }} \text { or, }
$$

\section{Actuator volume $=v \times P D /$ Actuator Continuous Power Density}

The lower volume of the two is used. Recall that actuator continuous power density is continuous specific power multiplied by density. The mass does not account for electrolyte, contacts, amplification mechanisms and the like, but only the mass of the active material itself. 
The 'battery' or power source mass (Y-axis) required to perform the work for the desired number of cycles in a given actuator is the greater of:

1. Total work divided by efficiency and specific energy, $S E$, of the power source and,

2. The power needed, $P$, divided by power source's specific power, $S P$ and efficiency.

\subsection{Results Table}

Figure 3 shows the summary table that is generated by the tool. Currently all actuators in the database are listed. Actuators that are struck out have been rejected because they do not have sufficient power per unit volume. At the time of writing this article, this is the only criterion used. The actuators are ordered by work density, and the numbers correspond to their labels on the plots. At present when the cursor is placed over the data points a number appears corresponding to the actuator number in the table. The data points can be selected with the cursor to produce a description of the actuator. Power density, work density, cycles and voltage columns are taken from the data table (Table 1). Actuator volume, mass and the relative frequency are determined for each actuator as described above.

\subsection{Further Information}

Further information on each actuator can be obtained by selecting the data points and columns associated with a particular actuator. A qualitative comparison chart is also provided, which can be selected under the heading "other resources". This additional information is intended to help designers make further selections based on criteria not considered in the web page, including temperature effects, cost, methods of activation and so on.

\section{APPLICATION}

The numbers entered in the text boxes in figure 1 are selected to be representative of the requirements for creating an artificial insect the size of a dragonfly. In order to mimic the dragonfly the mechanical power out is approximately 40 $\mathrm{mW}$. The wings beat at $40 \mathrm{~Hz}$, so the work per stroke is about $1 \mathrm{~mJ}$. In a dragonfly approximately half the total mass is consumed by muscle, suggesting that $150 \mathrm{mg}$ is allowable. However, since at present the selection is by volume and not mass, the approximate maximum dimensions that the muscle can consume are input, namely $l=40 \mathrm{~mm}, w=3 \mathrm{~mm}$, and depth $d=1 \mathrm{~mm}$. If the actuator is to survive for at least one hour of flying time then the cycle number is approximately 160,000. Low voltage is preferred since at present the most widely available portable electrical power sources (batteries, fuel cells and super-capacitors) are low in voltage. The numbers related to the power source are approximately those of a lead acid battery.

Graph 1 in Figure 2 indicates that most of the actuators are capable of performing the work required in this example. It is power that separates them (graph 2), with shape memory alloys, ferroelectric polymers, magnetic shape memory alloys and the two dielectric elastomers (VHB and silicone) exceeding the minimum requirements. A look at the hyperlinked text related to magnetic shape memory alloys however shows that this technology is likely ruled out due to the mass and volume needed to generate the magnetic fields needed in activation.

Graph 4 shows that battery mass is a real obstacle. Low efficiency actuators such as shape memory alloys (actuator 1) will consume far too much battery $(\sim 100 \mathrm{~g})$ to be seriously considered for more than very short duration flights. This leaves ferroelectric polymers and dielectric elastomers as the two remaining candidates. The higher efficiency actuators still consume several grams of battery. A higher energy density power source is required. Zinc air batteries (approximately $1 \mathrm{MJ} / \mathrm{kg}$ ) or micro fuel cells, need to be considered, and flight time may need to be sacrificed.

Graph 2 shows that mechanical amplification will be required for the ferroelectric polymers to work, with an amplification factor of at least 6 needed. The advantage of the dielectric elastomers is that relatively little mechanical amplification is needed. Both of these technologies are high voltage at present, and therefore will require step-up converters if they are to be powered by batteries or fuel cells. 


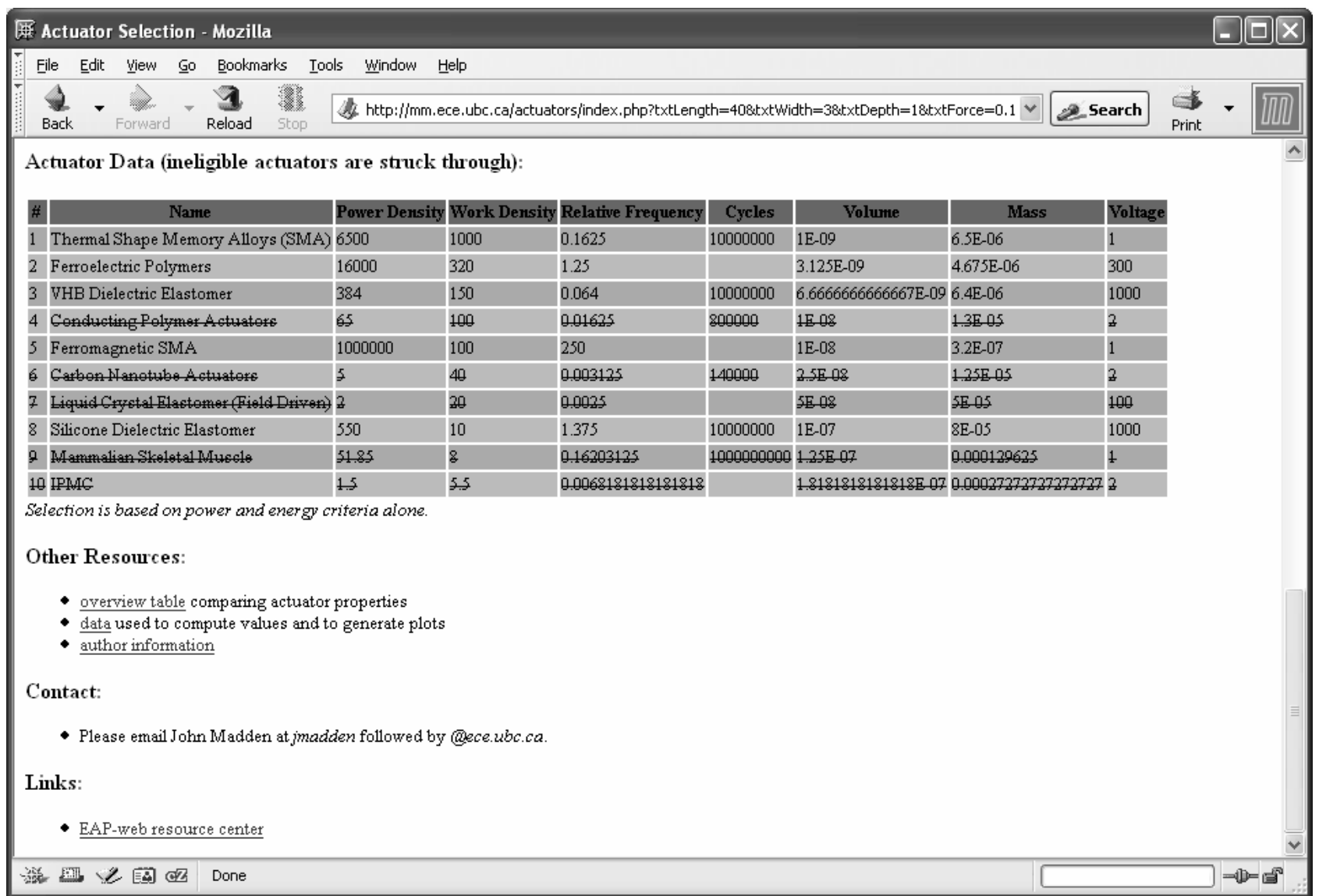

Figure 3: Results summary table and links to further information.

\section{CONCLUSIONS}

A web tool has been created that allows linear actuator technologies to be compared and evaluated based on volume, mass, work, power and power source requirements. An example is shown in which the number of candidate actuators is narrowed down via the selection criteria. Links are provided to actuator descriptions and further literature, enabling additional selection criteria to be used. It is hoped that this tool will encourage the adoption of new actuator technologies in applications where they provide the greatest benefit.

\section{REFERENCES}

1. J. Hollerbach, I. W. Hunter, J. Ballantyne, in The Robotics Review 2, O. Khatib, J. Craig, Lozano-Perez, Eds. (MIT Press, Cambridge, MA, 1992).

2. Hunter, I. W. and Lafontaine, S. A comparison of muscle with artificial actuators. Technical Digest IEEE Solid State Sensors and Actuators Workshop. 178-185. 92. IEEE.

3. Madden, J.D.W., Schmid,B., Hechinger,M., Lafontaine,S.R., Madden,P.G. A., Hover,F.S., Kimball,R., \& Hunter,I.W. Application of polypyrrole actuators: feasibility of variable camber foils. IEEE J. of Oceanic Engineering, 29(3), 738-749. 2004.

4. Madden,J.D.W., Vandesteeg,N.A., Anquetil,P.A., Madden,P.G.A., Takshi,A., Pytel,R.Z., Lafontaine,S.R., Wieringa,P.A., Hunter, I. W. Artificial muscle technology: physical principles and naval prospects. IEEE J. of Oceanic Engineering, 29(3), 706-728. 2004.

5. Madden, John D. Actuator selection for variable camber foils. Smart Structures and Materials 2004: Electroactive Polymer Actuators and Devices (EAPAD). 5385, 442-448. 2004-. San Diego, CA, USA, SPIE.

6. Sommer-Larsen, Peter and Kornbluh, Roy. Polymer Actuators. ACTUATOR 2002. Germany.

7. Wax, Steven G. and Sands, Randall R. Electroactive polymer actuators and devices. Smart Structures and Materials 1999:

Electroactive Polymer Actuators and Devices. 3669, 2-10. 99. Newport Beach, CA, USA, SPIE.

8. Electroactive Polymer (EAP) Actuators as Artificial Muscle, Yoseph Bar-Cohen, Ed. (SPIE, Bellingham, WA, 2001).

9. Zupan, M., Ashby, M. F., and Fleck, N. A. Actuator classification and selection - the development of a database. Advanced

Engineering Materials 4(12), 933-940. 2002. 